\title{
EL ROL DE LA ESTRATEGIA Y LA ESTRUCTURA ORGANIZACIONAL EN LA INNOVACIÓN NO I+D
}

\author{
(iD Lucy Carolina Elizalde-Bobadilla ${ }^{1}$, (D) Francisca Rojas-Santoyo ${ }^{2}$, ${ }_{\text {Rafael Leonardo Ochoa-Urrego }}{ }^{3}$

\begin{abstract}
${ }^{1}$ Administradora de Empresas, Especialista en Gestión de Portafolio y Valoración de Empresas y Magister en Gestión de Organizaciones. Profesora de Tiempo Completo en el Departamento de Administración de Empresas, Universidad Central. Bogotá, Colombia. lelizaldeb@ucentral.edu.co.

${ }^{2}$ Ingeniera de Sistemas, magister en Administración y Candidata a Doctora en Ingeniería Industria y Organizaciones de la Universidad Nacional de Colombia. Coordinadora Académica de Posgrados y docente en la Facultad de Contaduría Pública, Universidad Externado de Colombia.Bogotá, Colombia. francisca.rojas@uexternado.edu.co.

${ }^{3}$ Ingeniero de Sistemas de la Universidad Nacional de Colombia. Magister en Administración y Máster en e-Business: telecomunicaciones y nuevos modelos de negocio - Universidad de Cantabria en España. Doctor en Ingeniería - Industria y Organizaciones de la Universidad Nacional de Colombia. Posdoctor en Ciencias Políticas y Sociales - UNAM. Profesor Asociado en el Departamento de Contaduría Pública, Universidad Central. Bogotá,Colombia.rochoau@ucentral.edu.co.
\end{abstract}

\begin{abstract}
RESUMEN
Objetivo: el presente estudio analiza la relación de la estrategia y la estructura organizacional en el marco de la innovación no I+D.

Metodología: se realizó una revisión sistemática de la literatura en la que se identificaron 64 artículos de las bases de datos Scopus, Emerald y Proquest.

Originalidad: el presente estudio evidencia un conjunto de características, tanto de las organizaciones como del entorno, que contribuyen a la aparición de ANID y resultados de INID.

Resultados principales: se determinaron 24 factores que potencian, condicionan o resultan de este tipo de innovación. Contribuciones teóricas: como parte de los hallazgos se destaca la identificación de estrategias puntuales, la importancia de la capacidad de absorción para el entendimiento del fenómeno de innovación no I+D y el hecho de que las innovaciones más comunes bajo este enfoque son de tipo mercadeo y organizacional.
\end{abstract}

Palabras clave: Innovación no I+D; Estrategia organizacional; Estructura organizacional; Actividades de no $\mathrm{I}+\mathrm{D}$.

\section{THE ROLE OF STRATEGY AND ORGANIZATIONAL STRUCTURE IN NON-R\&D INNOVATION}

\section{ABSTRACT}

Objective: this article analyzes the relationship between strategy and organizational structure in the framework of non-R\&D innovation.

Methodology: a systematic review of literature was carried out in which 64 articles from Scopus, Emerald and Proquest databases were identified.

Originality: this study evidences a group of characteristics of the organizations and its sorroundings that contribute to the appearance of non-R\&D activities and results of nonR\&D innovation.

Main results: 24 factors that enhance, condition or result from this type of innovation were determined.
Theoretical contributions: as part of the findings, there stand out the identification of specific strategies, the importance of absorption capacity for understanding the phenomenon of non-R\&D innovation, and the fact that most common innovations under this approach are marketing and organizational.

Keywords: Non-R\&D innovation; Organizational strategy; Organizational structure; Activities of non-R\&D.

\section{O PAPEL DA ESTRATÉGIA E ESTRUTURA ORGANIZACIONAL NA INOVAÇÃO NÃO-P\&D RESUMO}

Objetivo: este estudo analisa a relação entre estratégia e estrutura organizacional no âmbito da inovação não-P\&D.

Metodologia: foi realizada uma revisão sistemática da literatura, na qual foram identificados 64 artigos das bases de dados Scopus, Emerald e Proquest.

Originalidade: o presente estudo evidencia um conjunto de características, tanto das organizações quanto do meio ambiente, que contribuem para o surgimento dos resultados do ANID e do INID.

Principais resultados: foram determinados 24 fatores que melhoram, condicionam ou resultam desse tipo de inovação. Contribuições teóricas: como parte dos resultados, destacam-se a identificação de estratégias específicas, a importância da capacidade de absorção para a compreensão do fenômeno da inovação não-P\&D e o fato de que as inovações mais comuns sob essa abordagem são do tipo marketing. e organizacional.

Palavras-chave: Inovação não-P\&D; Estratégia organizacional; Estrutura organizacional; Atividades nãoP\&D. 


\section{Introducción}

Desde el punto de vista tradicional, la innovación se concibe como el resultado de actividades asociadas a investigación y desarrollo (I+D) (Cohen, 2010; Qingqing et al., 2016; Rothwell, 1994). Esta perspectiva presupone la existencia de un proceso formal que incluye una serie de acciones específicas y predefinidas para generar innovaciones (Barge-Gil et al., 2011). Estos supuestos dejan por fuera del fenómeno innovador a empresas que, por su naturaleza, tamaño y capacidades económicas, no cuentan con recursos suficientes para tener un departamento propio o subcontratado de I+D. Dichas empresas incluso llegan a operar con procesos de innovación de carácter informal (Hobday, 2005). Esta no es una omisión menor si se tiene en cuenta que, por ejemplo, las organizaciones pequeñas y medianas no solo absorben cerca del $67 \%$ de la fuerza laboral a nivel mundial, sino que además representan entre el $60 \%$ y el 70\% del producto interno bruto (PIB) (Oficina Internacional del Trabajo, 2015).

Para dar explicación a los resultados de innovación de aquellas empresas que carecen de estructuras formales de $\mathrm{I}+\mathrm{D}$, han surgido estudios de orden teórico y empírico que acuñan los conceptos de innovación no I+D (INID) y de actividades no I+D (ANID). Bajo esta perspectiva, a través del enfoque de INID, las organizaciones sin estructura formal de I+D tienen la capacidad de definir acciones para mejorar su desempeño en el largo plazo, teniendo como insumo principal el entendimiento y la gestión consciente de sus propias capacidades de innovación (Guo et al., 2017). Específicamente, la gestión consciente de dichas capacidades se operacionaliza a través de una estrategia (López \& Mariño Arévalo, 2010), que, luego de ser definida o transformada, apoyaría la consolidación de una estructura que permita a la organización operar de manera eficiente y atender a los cambios del entorno (Chandler, 1990).

A pesar del avance que se ha realizado en el estudio de la INID, aún no es clara la relación entre los elementos propios de la estrategia y la estructura organizacional con la realización de ANID y el desempeño innovador basado en la INID. Por esta razón, con el objetivo de comprender el fenómeno de la INID se realizó una revisión sistemática de la literatura para caracterizar los factores asociados a la estrategia y la estructura organizacional que potencian o condicionan la INID.

La revisión de la literatura se complementó con un análisis de redes (Brandes \& Erlebach, 2005) en el que se identificaron las principales relaciones entre los factores asociados a la estrategia y la estructura organizacional y el desempeño organizacional en términos de la INID. Los principales resultados apuntan a que uno de los elementos más importantes es el desarrollo de la capacidad de absorción en las organizaciones. Asimismo, se encontró que como resultados de la INID se desarrollan mayormente innovaciones de procesos de negocio, específicamente en actividades de mercadeo y procesos de gestión organizacional.

Los resultados encontrados se exponen de la siguiente manera: en la primera sección se hace un breve recorrido sobre las bases teóricas de la INID. En la segunda sección se expone la metodología utilizada para la selección y análisis de los documentos científicos que evidencian el fenómeno de INID 
y las dimensiones seleccionadas para el presente estudio (estrategia y estructura). En la tercera sección se muestra un análisis de redes sobre las variables o factores organizacionales que influyen en la INID — con énfasis en aquellos que se consideran más relevantes- y una conceptualización de los factores seleccionados — con énfasis en su relación con la INID—. Por último, se presentan las conclusiones más destacadas del estudio.

\section{Marco teórico y antecedentes}

La innovación no I+D ha sido un concepto referenciado de manera tangencial en la literatura desde los años 80 con el trabajo de Kline \& Rosenberg (1986), quienes reconocieron que la innovación no necesariamente dependía del desarrollo de I+D (Arundel et al., 2008). Sin embargo, en años recientes se ha evidenciado un mayor interés por el estudio de esta temática como una visión alternativa y/o complementaria a la innovación tradicional (Qingqing et al., 2016); en concreto, cerca del 50\% de la bibliografía que menciona el término ha sido publicada en los últimos cinco años.

Al analizar la información de esta bibliografía se encuentran algunos aspectos en común, tales como la metodología aplicada (estudio de encuestas de innovación preexistentes y estudio de organizaciones concretas) y el contenido de las propuestas marco sobre los elementos concebidos dentro de la innovación no I+D (Diukanova \& López-Rodríguez, 2014; Hervas-Oliver et al., 2015; Moilanen et al., 2014). En este sentido, llama la atención que en los años más recientes los estudios sobre INID han trascendido Europa (área predominante en publicaciones sobre el tema) y han surgido estudios en el continente asiático (Guo et al., 2017; Lee \& Walsh, 2016).

Teniendo en cuenta que hasta el momento no existe una conceptualización formal de la INID y que el abordaje se ha centrado en identificar las ANID (Hou et al., 2018), la INID se puede definir como la innovación que se realiza a través de actividades que no cumplen de manera simultánea los cinco criterios señalados por el Manual de Frascati: novedad, creatividad, sistematicidad, transferibilidad y/o reproducibilidad e incertidumbre (Organización de Cooperación y Desarrollo Económicos [OECD], 2015). En este sentido, al revisar algunas ANID como la imitación, el diseño o la adopción tecnológica (Yanting et al., 2016), entre otras, es claro que el criterio de incertidumbre propuesto por la Organización de Cooperación y Desarrollo Económicos no se cumple. Este hecho se debe a que al usar un conocimiento preexistente se reducen las brechas de información sobre "los costos, o el tiempo, necesarios para lograr los resultados esperados" (OECD, 2015, p. 47), lo que a la postre hace que organizaciones con menores recursos puedan concretar innovaciones sin tener como punto de partida grandes inversiones.

Lo anterior supone una perspectiva interesante, para comprender los fenómenos de innovación en otros contextos, pero que aún presenta un estado incipiente en términos de investigación (Hou et al., 2018). 


\section{Metodología}

El presente trabajo se organiza en tres grandes etapas que se presentan a continuación.

\section{Primera etapa: revisión de la literatura}

Para el desarrollo de esta etapa se aplicaron de manera simultánea tres ecuaciones de búsqueda en las bases de datos Scopus, Emerald y Proquest. La búsqueda se realizó por título y palabras clave entre el 1 y el 12 de febrero de 2018. Luego de realizar las búsquedas independientes, se procedió a descartar los documentos duplicados. Los resultados obtenidos con cada una de las ecuaciones se presentan en la tabla 1 .

Tabla 1 - Resumen de la aplicación de las ecuaciones de búsqueda

\begin{tabular}{|c|c|c|}
\hline Ecuación de búsqueda & Base de datos & N..$^{\circ}$ de resultados \\
\hline \multirow{3}{*}{ "Non $R \& D "$ AND "strategy" } & Emerald & 73 \\
\hline & Scopus & 11 \\
\hline & Proquest & 10 \\
\hline \multirow{3}{*}{ "Non R\&D" AND "structure" } & Emerald & 69 \\
\hline & Scopus & 6 \\
\hline & Proquest & 6 \\
\hline \multirow{3}{*}{ "Non R\&D" AND "organizational structure" } & Emerald & 0 \\
\hline & Scopus & 0 \\
\hline & Proquest & 1 \\
\hline
\end{tabular}

Fuente: Elaboración propia.

Con el fin de evaluar la pertinencia de los artículos obtenidos en las búsquedas, se diseñaron los criterios de inclusión y exclusión mostrados en la tabla 2.

Tabla 2 - Criterios de inclusión y exclusión

\begin{tabular}{|l|l|}
\hline \multicolumn{1}{|c|}{ Filtro } & \multicolumn{1}{|c|}{ Critério } \\
\hline 1 & Incluir solo fuentes arbitradas \\
\hline 2 & Revisión del abstract y el título \\
\hline 3 & Disponible para su descarga \\
\hline 4 & Documentos que presenten evidencia empírica del estudio de la INID \\
\hline
\end{tabular}

Fuente: Elaboración propia. 
Como resultado de la aplicación de los criterios de inclusión, se seleccionaron 64 artículos clasificados como señala la tabla 3.

Tabla 3 - Clasificación de los artículos seleccionados

\begin{tabular}{|l|l|c|}
\hline \multicolumn{1}{|c|}{ Dimensión } & \multicolumn{1}{|c|}{ Base de datos } & N. ${ }^{\mathbf{0}}$ de artículos \\
\hline \multirow{3}{*}{ Estrategia } & Emerald & 12 \\
\cline { 2 - 3 } & Scopus & 27 \\
\cline { 2 - 3 } & Proquest & $\mathbf{4 6}$ \\
\hline \multirow{2}{*}{ Subtotal } & Emerald & 11 \\
\hline \multirow{3}{*}{ Estructura } & Scopus & 6 \\
\cline { 2 - 3 } & Proquest & 1 \\
\hline Subtotal & & $\mathbf{1 8}$ \\
\hline Total & & $\mathbf{6 4}$ \\
\hline
\end{tabular}

Fuente: Elaboración propia.

\section{Segunda etapa: análisis de redes}

El objetivo de esta etapa fue identificar los factores más relevantes asociados a la INID y, al mismo tiempo, analizar las relaciones que tienen dichos factores con la INID en términos de la magnitud de esta relación y su dirección. En primer lugar, se analizaron los 64 artículos seleccionados con el fin de recopilar los factores considerados como influenciadores o resultantes de la INID. En el presente trabajo se entendió como factores aquellas características o decisiones organizacionales que tengan alguna relación con la realización de actividades de INID o con los resultados de dichas actividades. Como resultado, se identificaron los factores con una relación verificable y con evidencias tanto empíricas como teóricas. Al final del análisis se establecieron 129 factores que fueron agrupados en tres categorías: factores generales, factores asociados a la estructura y factores asociados a la estrategia.

Seguidamente, se construyó la red de relaciones de los factores mediante pares de elementos cuya relación hubiese sido verificada en la literatura analizada. Cada relación estaba constituida por un factor asociado a la estrategia o la estructura organizacional y la INID. En otras palabras, se identificaron aquellos factores organizacionales que hubiesen tenido una relación directa y verificada con la INID. Después, se le asignó un peso a cada una de estas relaciones, para lo cual se tomó como métrica el número de artículos que verificaban la relación entre los factores identificados y la INID. Asimismo, se reconoció la dirección de la relación; es decir, se identificó si el factor condiciona o potencia la innovación o si la innovación es la que genera un efecto sobre el factor. Como resultado se obtuvieron 262 relaciones, de las cuales se seleccionaron aquellas que tuvieran un peso superior a 5. De esta manera 
quedaron 24 relaciones priorizadas, que están constituidas por 24 factores asociados a la estrategia o la estructura organizacional. Para la construcción de la red de grafos se utilizó el software Gephi.

Tercera etapa: análisis y descripción de los factores y las relaciones

Con el fin de profundizar en los conceptos y variables identificadas se realizó la conceptualización de los 24 factores y sus relaciones. En esta etapa se construyó la definición de cada uno de los conceptos y se describió la relación que tienen con la INID según la literatura seleccionada.

\section{Resultados}

Los resultados se presentan en dos secciones: la primera se concentra en analizar la red de conceptos creada, mientras que la segunda examina los factores más relevantes de cada familia de factores.

\section{Análisis de la red de conceptos}

Luego de seleccionar los factores más relevantes según las métricas diseñadas, se configuró un grafo de relaciones que muestra los factores identificados dentro de las tres familias de factores diseñadas (cada una con un color diferente). Igualmente, se muestran las relaciones y el peso relativo de estas con respecto a los demás factores de la muestra. Para expresar estas variables de análisis, el grafo utiliza las cabezas de flecha (para identificar la dirección de la relación) y el grosor de la línea que une los dos factores (para identificar el peso relativo de la relación). Asimismo, se agrupan los factores de acuerdo con la dimensión asociada: los 3 generales se muestran en azul, los 12 asociados a la estrategia aparecen en rojo, los 4 asociados a la estructura organizacional están en amarillo y los 4 de resultados de innovación se presentan en verde. Es importante resaltar que se presentaron dos relaciones entre elementos de dos dimensiones distintas que se marcan en verde oliva (ubicada a la izquierda del grafo) y en gris (ubicada a la derecha del grafo). Los 23 factores anteriormente relacionados y el factor de la INID completan los 24 factores del modelo. La red resultado del análisis se muestra en la figura 1. 
Figura 1 - Red de factores de innovación no I+D y sus relaciones

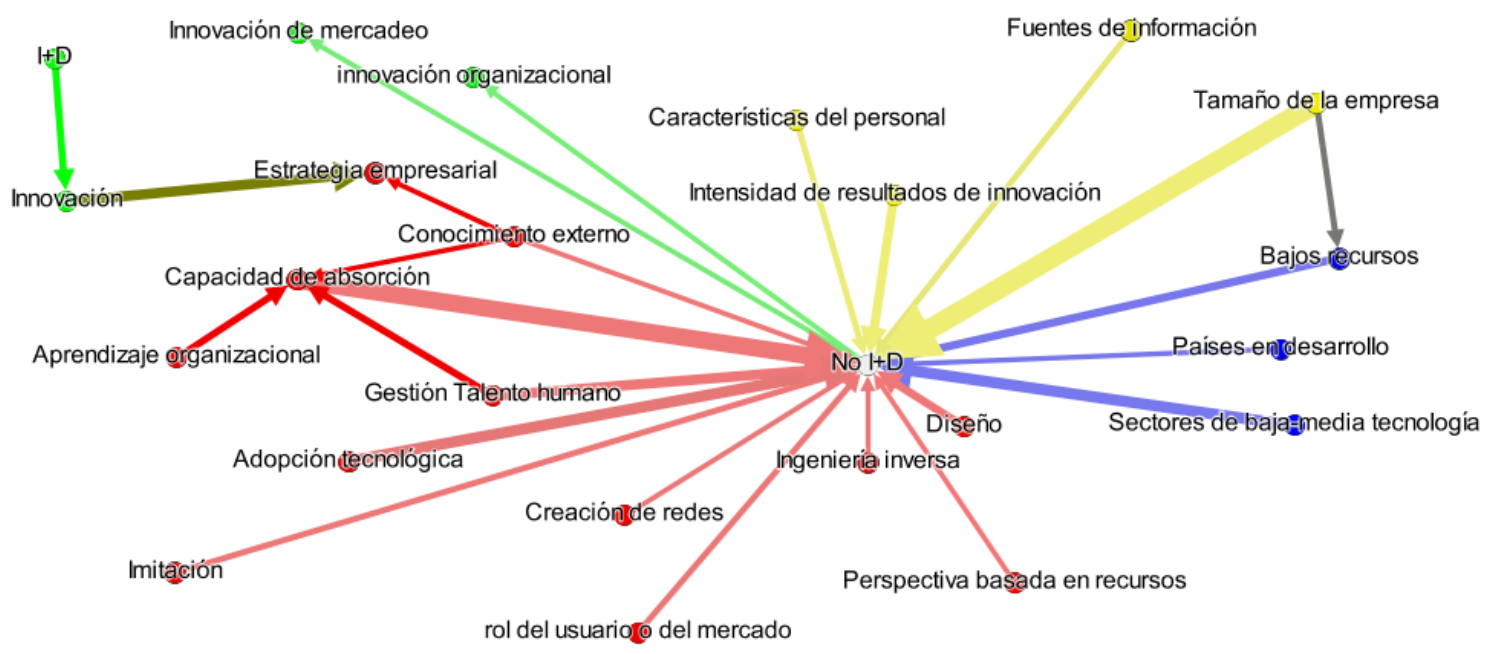

Fuente: Elaboración propia con resultados del software Gephi.

El elemento que más resalta en la figura 1 es el tamaño de la relación entre el factor de capacidad de absorción y la INID. Esto obedece al hecho de que distintas perspectivas teóricas se apoyan en la teoría de capacidad de absorción para explicar el funcionamiento de la INID, (Guo et al., 2017; Moilanen et al., 2014; Petti \& Zhang, 2016; Qingqing et al., 2016; Trujillo-Ruiz et al., 2015; Ueki, 2017). Sobre el mismo factor de capacidad de absorción se observa un pequeño clúster asociado con otros tres factores: gestión del talento humano, aprendizaje organizacional y conocimiento externo. Otro elemento relevante en esta figura es la conexión indirecta del concepto I+D con la INID, ya que se reconoce la posible complementariedad existente entre las actividades asociadas a I+D y las ANID (Santamaría et al., 2009; Siddharthan \& Agarwal, 1992).

\section{Conceptualización de factores}

A continuación se conceptualizan los distintos factores de acuerdo con las dimensiones.

\section{Bajos recursos tecnológicos y económicos como factores para la aparición de la INID}

Esta dimensión agrupa factores que representan características compartidas por las organizaciones que en general desarrollan INID. Los factores asociados son: países en desarrollo, sectores de baja-media tecnología y bajos recursos.

En general, la literatura ha logrado demostrar que en países en desarrollo los resultados de innovación obtenidos por las organizaciones están más vinculados a la INID que a la visión tradicional fundamentada en I+D. Como ejemplos se encuentran los análisis empíricos realizados en países 
particulares como China, Laos, Tailandia y Vietnam (Petti \& Zhang, 2016; Ueki, 2017) y la comparación entre países desarrollados y países en desarrollo realizada por Huang et al. (2010), quienes específicamente mencionan el rol de la INID en Bulgaria, Rumania y Estonia. Los autores mencionados coinciden en afirmar que la mayor incidencia de INID obedece a que los países menos desarrollados cuentan con una infraestructura de innovación menos avanzada.

Por su parte, según Arundel et al. (2008) y Kirner \& Som (2015a), los sectores de baja-media tecnología orientan sus esfuerzos principalmente a desarrollar de forma incremental tecnologías existentes y, por tanto, las ANID tienen un rol importante al interior de este tipo de organizaciones. En estudios empíricos de organizaciones de este tipo se ha encontrado que tienen fuertes niveles de innovación, a pesar de no contar con actividades formales de I+D (Qingqing et al., 2016; Yanting et al., 2016).

Por otro lado, la asociación de la INID con los bajos recursos se debe a que la innovación tradicional a través de $\mathrm{I}+\mathrm{D}$ presenta altos niveles de riesgo e importantes inversiones que generan rendimientos a muy largo plazo (Rammer et al., 2009). Esto dificulta la realización de este tipo de inversiones por organizaciones cuyo panorama de acción es de más corto plazo.

\section{La INID como estrategia o la estrategia para la INID}

Dentro de la dimensión de estrategia se identificaron doce factores: estrategia organizacional, capacidad de absorción, conocimiento externo, perspectiva basada en recursos, gestión del talento humano, aprendizaje organizacional, diseño, rol del usuario o del mercado, creación de redes, adopción tecnológica, imitación e ingeniería inversa. Estos tres últimos se analizarán de manera conjunta, tomando como referencia la categoría denominada por Qingqing et al. (2016) como "actividades técnicas de innovación no basadas en I+D” (p. 1066).

Como ya se mencionó, la relación con más peso fue la encontrada entre la capacidad de absorción y la INID. Esta capacidad es entendida como "un conjunto de rutinas y procesos organizacionales por los cuales la firma adquiere, asimila, transforma y explota conocimiento" (Zahra \& George, 2002, p. 186). De acuerdo con Som (2012), la capacidad de absorción es un factor que cumple un papel primordial en las organizaciones que usan de manera intensiva la INID. Esta importancia nace, entre otros elementos, de la relación que existe entre esta capacidad y la comunicación y coordinación con organizaciones aliadas.

Adicionalmente, gracias a la capacidad de absorción, la organización mejora la relación que tiene con su entorno, lo que genera un aumento en el acceso a los diferentes tipos de conocimiento externo (segundo factor). Este conocimiento sirve para suplir y complementar su propio conocimiento y sus recursos internos (Sempere-Ripoll \& Hervás-Oliver, 2002) y, como consecuencia, le permite a la organización realizar una mayor actividad innovadora (Bogers \& Lhuillery, 2011; Flor et al., 2011; 
González-Campo \& Hurtado, 2014; Hervas-Oliveret al., 2012; Hervas-Oliver et al., 2011). Para ello, las empresas pueden elegir entre una variedad de estrategias (Bogers \& Lhuillery, 2011), dentro de las que se cuenta la obtención de beneficios generados por derrames tecnológicos (spillovers), que sería en términos de Henry Mintzberg, una estrategia emergente. Por otra parte, las organizaciones pueden optar por una estrategia deliberada, en la que se definan explícitamente mecanismos para la adquisición y el aprovechamiento del conocimiento externo.

Por medio de estas estrategias, la capacidad de absorción proporciona conocimiento con fines tecnológicos o de innovación y desarrollo. Además, potencia el proceso de mejoramiento de áreas no dedicadas a I+D como mercadeo y producción, en las cuales también es posible desarrollar innovación a partir del conocimiento recibido de clientes y proveedores.

Otra teoría identificada como factor de INID es la perspectiva basada en recursos. De acuerdo con Barney (1991), esta perspectiva tiene como premisa que las diferencias de desempeño entre las organizaciones subyacen a su propia configuración de activos, capacidades, procesos y conocimiento; bajo esa configuración única cada organización implementa sus propias estrategias. Como consecuencia, y relacionando esta perspectiva con la INID, las organizaciones pueden desarrollar innovación organizacional y en mercadeo mediante la gestión de sus propias capacidades, lo cual les permite "reforzar la innovación tecnológica y contrarrestar su falta de capacidades de I+D" (Hervas-Oliveret al., 2015, p. 33).

Como cuarto factor estratégico que fortalece el desarrollo de INID se encuentra la gestión del talento humano. En esta relación se hace evidente que las organizaciones que no cuentan con I+D tienen la capacidad de generar innovaciones al aplicar herramientas como trabajo en equipo, mecanismos facilitadores de acuerdos de cooperación para acceder al conocimiento externo, métodos de reclutamiento para identificar a las personas adecuadas para promover la innovación, capacitación para la innovación, sistemas de recompensa y herramientas de desarrollo profesional, entre otros (Rammer et al., 2009).

Por su parte, el aprendizaje organizacional se presenta, según Griffin \& Somermeyer (2007), cuando "una organización que continuamente prueba y actualiza la experiencia de sus miembros y transforma esa experiencia en mejores procesos de trabajo y en conocimiento que es accesible para toda la organización" (p. 486). Es una variable que encuentra una alta compatibilidad con la perspectiva de INID, ya que ambas se basan en rutinas para generar los cambios al interior de la organización. A su vez, cuando la organización realiza ANID, como es el caso de las modificaciones menores sobre productos o servicios existentes, amplía su experiencia y, por consiguiente, activa posibilidades de aprendizaje organizacional (Guo et al., 2017).

La variable diseño permite el desarrollo y la mejora de los productos existentes, por ejemplo, con herramientas de software para diseño asistido CAD-CAM. Diferentes estudios reconocen las actividades de diseño como un mecanismo que ayuda a mejorar la capacidad innovadora de una empresa (Hervas-Oliver et al., 2011, 2012; Santamaría et al., 2009). Si bien el diseño es reconocido como una 
fuente de innovación estética dentro de las innovaciones de mercado, en las mediciones tradicionales de innovación todavía se descarta como una fuente de innovación de productos a menos que influya en las funciones y/o usos del producto (Filipa Soares Passos Cardoso \& Torkkeli, 2014).

En el caso del rol del usuario, su importancia radica en la generación de INID, ya que, a partir de sus demandas o especificaciones, las empresas pueden introducir innovaciones. Bajo esta perspectiva se reconoce el rol del usuario como un direccionador de innovación tan importante como la I+D (Kastelli et al., 2016). Otros autores afirman que además de las consideraciones tecnológicas, las empresas deben tener una orientación hacia el mercado (Filipa Soares Passos Cardoso \& Torkkeli, 2014; Kirner \& Som, 2015) para obtener un mayor conocimiento del entorno como propulsor de la innovación (Bogers \& Lhuillery, 2011).

Por su parte, el factor de creación de redes es importante porque a través de ellas se crean rutas privilegiadas para que empresas de baja y media tecnología accedan, creen, recombinen e integren nuevos conocimientos de fuentes externas (Hervas-Oliver et al., 2012; Rammer et al., 2009). Esto conlleva la generación de enlaces dentro de la misma organización y el trabajo con actores externos; por ejemplo, es posible desarrollar proyectos de cooperación para que los costos, los riesgos y la incertidumbre asociados a un proceso de innovación puedan ser compartidos y minimizados (Filipa Soares Passos Cardoso \& Torkkeli, 2014). De allí que se haya motivado el crecimiento en el uso de las redes externas y simultáneamente se produzca un descenso en el número de departamentos de $\mathrm{I}+\mathrm{D}$ interna (Sempere-Ripoll \& Hervás-Oliver, 2011).

La adopción de tecnología, la imitación y la ingeniería inversa son consideradas como métodos para desarrollar INID. Mientras que el primero implica la obtención de productos, procesos o innovaciones organizacionales preexistentes que requieren poco trabajo adicional para su implementación, los segundos conllevan un mayor nivel de creatividad para la reproducción de productos y procesos ya establecidos (Arundel et al., 2008). El componente común de los tres métodos es el aprovechamiento de conocimiento, lo cual es coherente con la perspectiva de la INID y otro factor identificado como relevante: la capacidad de absorción (Qingqing et al., 2016).

\section{La estructura para la INID}

En la dimensión de la estructura se identificaron cuatro variables o factores como los más representativos: tamaño de la empresa, intensidad de los resultados de innovación, fuentes de información y características del personal. Tal como ocurre en el estudio de la innovación basada en I+D, el tamaño de la empresa se identifica como un elemento de alta relevancia para la generación de innovaciones. Este factor puede ser medido por la cantidad de empleados o colaboradores que están vinculados de manera directa con la organización (Trigo, 2013) o por el nivel de ventas reportadas en el último año (Sterlacchini, 1999). 
En la literatura no solo se considera que el tamaño de la organización puede ser una medida indirecta de las capacidades de innovación (Huang et al., 2010), sino que además se pone en evidencia una relación directa entre el tamaño de la empresa y la formalización de las acciones y procesos tendientes a la innovación. En primera instancia, las empresas de menor tamaño tienden a emplear más INID (Diukanova \& López-Rodríguez, 2014; Huang et al., 2010; Sterlacchini, 1999). Posteriormente, y a medida que las organizaciones crecen y ganan experiencia y especialización, tienden a realizar actividades formales de I+D (Veugelers \& Cassiman, 1998). En consecuencia, las empresas grandes son consideradas como desarrolladoras de conocimiento, mientras que las empresas pequeñas y medianas se orientan hacia la adopción de tecnologías (Trigo, 2013). Este comportamiento coincide con los hallazgos sobre la cantidad de recursos tecnológicos y financieros disponibles para la realización de actividades de innovación. De esta manera, la escasez de recursos incentiva a las empresas de menor tamaño a buscar alternativas generadoras de innovación de bajo riesgo, por lo que terminan dejando a un lado el enfoque I+D (Moilanen et al., 2014).

Como segundo factor, la intensidad de los resultados de innovación se entiende como la probabilidad de que una organización genere innovaciones (Trigo, 2013). Bajo la mirada de la INID, dicha medida implicaría el esfuerzo en la adquisición o compra de maquinaria o equipos innovadores, la adquisición de conocimiento externo o la colaboración con aliados, cuando dichas adquisiciones son vistas como una fuente de innovación (Som et al., 2013). En consecuencia, la intensidad de innovación a partir de ANID puede medirse como la tasa entre la inversión en actividades de mercadeo y las ventas (Doukas et al., 1999) o el gasto en la adquisición de patentes y licencias, entrenamiento de personal, investigación de mercados e inversión para ampliar o mejorar la capacidad de producción (Barge-Gil et al., 2011; Brouwer \& Kleinknecht, 1997).

Como tercer factor se identificaron las fuentes de información utilizadas para alimentar el proceso de innovación. Es importante resaltar que esto coincide con las habilidades asociadas a la capacidad de innovación explicadas anteriormente. En las referencias teóricas y empíricas se ha establecido que cuando la organización tiene a sus proveedores como fuente primaria de información para la identificación de oportunidades tecnológicas, tiende a realizar más ANID. Por el contrario, las organizaciones que tienen a centros de investigación y universidades como fuentes de información realizan más actividades de I+D (Huang et al., 2010). Este tipo de organizaciones que utilizan como fuente primaria a sus proveedores se denominan organizaciones dominadas por los proveedores (Som, 2012; Sterlacchini, 1999).

Por último, las características del personal encierran varios elementos entre los que se destacan el porcentaje de personas que han recibido educación avanzada (Huang et al., 2010; Moilanen et al., 2014), la experiencia del personal (Lee \& Walsh, 2016; Moilanen et al., 2014), la remuneración recibida (Pulak \& Bikash, 1997) y la tendencia a trabajar en equipo (Rammer et al., 2009). Así mismo, las organizaciones que utilizan ANID tienden a tener personal con menor nivel de formación y capacitación (Huang et al., 2010). Los empleados educados y con experiencia son requisito indispensable para la 
generación de procesos de innovación basados en I+D (Huang et al, 2010), mientras que los empleados involucrados en procesos de no I+D tienen a aprender más del día a día que mediante capacitaciones formales (Barge-Gil et al., 2011; Lee \& Walsh, 2016). Como consecuencia de su poca experiencia y sus niveles educativos, los empleados asociados a las ANID reciben remuneraciones más bajas que las ofrecidas a los empleados involucrados en actividades de I+D (Pulak \& Bikash, 1997).

Debido a su nivel de formación y alta orientación a tareas puntuales, los trabajadores de no I+D construyen información que puede generar procesos de innovación a partir de sus actividades y rutinas diarias (Lee \& Walsh, 2016). Esta visión coincide con lo encontrado para la variable de aprendizaje organizacional.

\section{La INID como generadora de innovaciones}

En cuanto a los resultados de la INID se identificaron cuatro factores. En el marco de los tipos de innovación definidos por el Manual de Oslo (Organización de Cooperación y Desarrollo Económicos, \& Oficina de Estadísticas de las Comunidades Europeas , 2005), se ha establecido que las innovaciones más comunes para las organizaciones que adoptan las estrategias basadas en ANID se concentran en la innovación de procesos de negocio, específicamente de tipo 3 (mercadeo) y 5 (administración y gestión) (Hervas-Oliver et al., 2015; Yanting et al., 2016), debido a los recursos empleados para estos tipos de innovación.

Para la innovación en mercadeo, Chen (2006) destaca la implementación de métodos o tecnologías que, luego de obtener y analizar información del cliente, permiten ofrecer mejores tarifas o reducir los costos de transacción. Estos procesos se pueden dar gracias a estrategias basadas en la capacidad de absorción o de adopción tecnológica. Dado el tamaño de las organizaciones que realizan ANID, es fundamental el papel que juega el establecimiento de relaciones con socios y la adquisición de información a través de proveedores, competidores, universidades o asociaciones (Yanting et al., 2016).

\section{Discusión}

La revisión de literatura en materia de INID y su relación con la gestión y la estructura organizacional permitieron la identificación, análisis y conceptualización de 23 factores que potencian, condicionan o resultan de este tipo de innovación. Si bien los factores mencionados no son los únicos, son los que más se referencian en las fuentes académicas consultadas. Así, por ejemplo, la cultura organizacional, que ha sido ampliamente reconocida como un aspecto fundamental para los procesos de innovación en general (Kahn et al., 2012; Markham \& Lee, 2013), no se referenció, pues para el caso específico de INID existen otros elementos más protagónicos. 
Esta revisión, también permitió identificar estrategias puntuales para la consecución de innovación a través de INID. Este es el caso de la gestión del talento humano, aprendizaje organizacional, diseño, creación de redes, adopción tecnológica, imitación e ingeniería inversa, estrategias que, en caso de ser adoptadas, deberían permitir la disposición de la estructura organizacional, teniendo como base que la estructura organizacional sigue a la estrategia (Chandler, 1990).

Con los resultados aportados por el presente estudio se evidencia un conjunto de características, tanto de las organizaciones como del entorno, que ayudan a la aparición de ANID y resultados de INID. Dichas características ya habían sido identificadas de manera aislada en los distintos estudios analizados. De esta manera, el principal aporte de esta investigación es ubicar en un solo escenario los factores asociados al entorno, la estrategia y la estructura organizacional en un ambiente donde prima la INID. Con el análisis integrador propuesto se busca abrir una línea de trabajo en la que la discusión y el análisis del comportamiento innovador de las pequeñas empresas se realicen a partir de la combinación de estos factores identificados para comprender de una mejor manera cómo se generan los resultados de innovación a partir de la ANID.

\section{Conclusiones}

Aunque el Manual de Oslo reconoce que la heterogeneidad de los contextos de los países puede dar lugar a adaptaciones sobre los parámetros dados desde la OCDE en materia de innovación (Organización de Cooperación y Desarrollo Económicos, \& Oficina de Estadísticas de las Comunidades Europeas , 2005), el enfoque dominante continúa siendo la innovación a través de procesos de I+D. Esta situación tiene consecuencias a nivel de la academia, la organización y la política pública.

En primera instancia, existe poca investigación alrededor de la INID; esto se demuestra en los resultados preliminares de la búsqueda realizada al comparar el volumen de producción académica referente al tema. En la literatura encontrada se observa un promedio de 16 documentos al año versus 3500 asociados al enfoque I+D para el periodo 2010-2018. Esto lo convierte en un campo de estudio aún incipiente que al desarrollarse ofrecería una perspectiva de innovación para los países en desarrollo, los sectores de baja y media tecnología y las empresas de menor tamaño (Rammer et al., 2009). Justamente en estos tres escenarios, la generación de innovación por vías que sean adaptadas al contexto y alternativas a las tradicionales cobra valor como medio para lograr mayores niveles de productividad e ingreso y así mejorar las condiciones de calidad de vida de la población.

En términos organizacionales, la concentración exclusiva en I+D no solo invisibiliza sus esfuerzos en materia de innovación, sino que además impide una gestión consciente mediante la definición de estrategias y su consecuente estructura para desarrollarlas. En este contexto específico, se vislumbra la oportunidad del entendimiento del fenómeno de la INID a través del concepto de la 
capacidad de absorción aplicado a organizaciones o sectores específicos, tal como se ha hecho en estudios empíricos en China, Laos, Tailandia y Vietnam (Petti \& Zhang, 2016; Ueki, 2017).

En cuanto a las repercusiones en política pública, se están dejando de incentivar actividades que permiten el desarrollo de capacidades innovadoras en organizaciones pequeñas y medianas y en sectores importantes de la economía (Santamaría et al., 2009a) que, si bien logran generar innovaciones, no necesariamente lo hacen recurriendo a actividades de $\mathrm{I}+\mathrm{D}$, precisamente por su naturaleza, su tamaño y su acceso limitado a recursos. Esta situación es particularmente importante en un país como Colombia, donde cerca del 95\% son microempresas (Ávila \& Rodríguez, 2017) y en donde la participación en el PIB de las industrias de baja tecnología es cercano al 26\% (World Bank, 2017).

\section{Referencias}

Arundel, A., Bordoy, C., \& Kanerva, M. (2008). Neglected innovators: how do innovative firms that do not perform R\&D innovate? Results of an analysis of the Innobarometer 2007 survey N. ${ }^{\circ}$ 215. INNO-Metrics Thematic Paper. Brussels: European Comission.

Ávila, R., \& Rodríguez, J. (2017). Estrategias Nacionales de Inclusión Financiera (NFIS): aplicación en las pymes colombianas. In Tamayo, C. E. \& Malagón, J. (Eds.), Ensayos sobre inclusión financiera en Colombia (pp. 257-286). Bogotá: Asobancaria, Banco Interamericano de Desarrollo.

Barge-Gil, A., Nieto, M. J., \& Santamaría, L. (2011). Hidden innovators: the role of nonR\&D activities. Technology Analysis and Strategic Management, 23(4): 415-432. https://doi.org/10.1080/09537325.2011.558400

Barney, J. (1991). Firm resources and sustained competitive advantage. Journal of Management, 17(1), 99-120. http://doi.org/gpm

Bogers, M., \& Lhuillery, S. (2011). A functional perspective on learning and innovation: investigating the organization of absorptive capacity. Industry \& Innovation, 18(6), 581610. https://doi.org/10.1080/13662716.2011.591972

Brandes, U., \& Erlebach, T. (Eds.). (2005). Network analysis: methodological foundations. Heidelberg: Springer-Verlag Berlin Heidelberg.

Brouwer, E., \& Kleinknecht, A. (1997). Measuring the unmeasurable: a country's non-R\&D expenditure on product and service innovation. Research Policy, 25(8): 1235-1242. https://doi.org/10.1016/S0048-7333(96)00902-X

Chandler, A. D. (1990). Strategy and structure. Chapters in the history of the industrial enterprise (2nd ed.). Cambridge, Massachusetts: MIT Press.

Chen, Y. (2006). Marketing innovation. Journal of Economics \& Management Strategy, 15(1): 101-123. https://doi.org/10.1111/j.1530-9134.2006.00093.x

Cohen, W. M. (2010). Fifty years of empirical studies of innovative activity and performance. In Hall, B. H., \& Rosenberg, N. (Eds.), Handbook of the economics of innovation (Vol. 1) (pp. 129-213). Amsterdam: Elsevier B. V. https://doi.org/10.1016/S0169-7218(10)01004-X

Diukanova, O., \& López-Rodríguez, J. (2014). Regional impacts of non-R\&D innovation expenditures across the EU regions: simulation results using the Rhomolo CGE Model. Investigaciones Regionales, 29: 91-111.

Doukas, J. Y., Pantzalis, C., \& Kim, S. (1999). Intangible assets and the network structure of MNCs. Journal of International Financial Management \& Accounting, 10(1): 1-23. https://doi.org/10.1111/1467-646X.00040

Filipa Soares Passos Cardoso, A., \& Torkkeli, M. (2014). Innovation in footwear companies does it pay off? Journal of Engineering, Design and Technology, 12(1): 128-154. 
https://doi.org/10.1108/JEDT-09-2010-0063

Flor, M. L., Oltra, M. J., \& García, C. (2011). La relación entre la capacidad de absorción del conocimiento externo y la estrategia empresarial: un análisis exploratorio. Revista Europea de Dirección y Economía de la Empresa, 20(1): 69-87. http://hdl.handle.net/10234/37426

González-Campo, C. H., \& Hurtado, A. (2014). Influencia de la capacidad de absorción sobre la innovación: un análisis empírico en las mipymes colombianas. Estudios Gerenciales, 30(132): 277-286. https://doi.org/10.1016/j.estger.2014.02.015

Griffin, A., \& Somermeyer, S. (2007). Appendix II: The PDMA Glossary for New Product Development. In The PDMA ToolBook 3 for new product development (pp. 465-508). Hoboken, New Jersey: John Wiley \& Sons. https://doi.org/10.1002/9780470209943.app2

Guo, Y., Chen, X., \& Zheng, G. (2017). How do non-R\&D-based innovations affect SMEs' performance? The mediating role of dynamic capabilities. In 2016 Portland International Conference on Management of Engineering and Technology (PICMET): Technology Management For Social Innovation (pp. 879-886). Piscataway, New Jersey: IEEE. https://doi.org/10.1109/PICMET.2016.7806643

Guo, Y., Zheng, G., \& Liu, F. (2017). Non-R\&D-based innovation activities and performance in Chinese SMEs: the role of absorptive capacity. Asian Journal of Technology Innovation, 25(1): 110-128. https://doi.org/10.1080/19761597.2017.1302548

Hervas-Oliver, J., Albors-Garrigos, J., \& Baixauli, J. (2012). Beyond R\&D activities: the determinants of firms' absorptive capacity explaining the access to scientific institutes in low-medium-tech contexts. Economics of Innovation and New Technology, 21(1): 55-81. http://doi.org/bj3gr2

Hervas-Oliver, J., Albors Garrigos, J., \& Gil-Pechuan, I. (2011). Making sense of innovation by R\&D and non-R\&D innovators in low technology contexts: a forgotten lesson for policymakers. Technovation, 31(9): 427-446.

https://doi.org/10.1016/j.technovation.2011.06.006

Hervas-Oliver, J. L., Sempere-Ripoll, F., Boronat-Moll, C., \& Rojas, R. (2015).

Technological innovation without R\&D: unfolding the extra gains of management innovations on technological performance. Technology Analysis and Strategic Management, 27(1): 19-38. https://doi.org/10.1080/09537325.2014.944147

Hirsch-Kreinsen, H. (2008). "Low-technology": a forgotten sector in innovation policy. Journal of Technology Management and Innovation, 3(3): 11-20. https://doi.org/10.4067/S0718-27242008000100002

Hobday, M. (2005). Firm-level innovation models: perspectives on research in developed and developing countries. Technology Analysis \& Strategic Management, 17(2): 121-146. https://doi.org/10.1080/09537320500088666

Hou, J., Chen, J., Song, H., \& Wang, G. (2018). Are non-R\&D innovation activities actually effective for innovation sustainability? Empirical study from Chinese high-tech industry. Sustainability, 11(1): 174. https://doi.org/10.3390/su11010174

Huang, C., Arundel, A., \& Hollanders, H. (2010). How firms innovate: R\&D, non R\&D, and technology adoption. UNU-MERIT Working Paper Series, 27: 146-146. https://www.merit.unu.edu/publications/working-papers/abstract/?id=3931

Kahn, K. B., Barczak, G., Nicholas, J., Ledwith, A., \& Perks, H. (2012). An examination of new product development best practice. Journal of Product Innovation Management, 29(2): 180-192. https://doi.org/10.1111/j.1540-5885.2011.00888.x

Kastelli, I., Tsakanikas, A., \& Caloghirou, Y. (2016). Technology transfer as a mechanism for dynamic transformation in the food sector. The Journal of Technology Transfer, 43(4), 882900. https://doi.org/10.1007/s10961-016-9530-3

Kim, L., \& Nelson, R. R. (2000). Introduction. In Kim, L., \& Nelson, R. (Eds.), Technology, learning and innovation: experiences of newly industrializing economies (pp. 1-10). 
Cambridge: Cambridge University Press.

Kline, S. J., \& Rosenberg, N. (1986). An overview of innovation. En Landau, R. \& Rosenberg, N. (Eds.), The positive sum strategy. Harnessing technology for economic growth (pp. 275-305). Washington D. C.: National Academy Press.

Lee, Y. N., \& Walsh, J. P. (2016). Inventing while you work: knowledge, non-R\&D learning and innovation. Research Policy, 45(1): 345-359.

https://doi.org/10.1016/j.respol.2015.09.009

López, P. A., \& Mariño Arévalo, A. (2010). Hacia una evolución en el campo del conocimiento de la disciplina administrativa: de la administración de empresas a la gestión de organizaciones. Revista Facultad de Ciencias Económicas: Investigación y Reflexión, 18(2): 75-95. https://doi.org/10.18359/rfce.2273

Markham, S. K., \& Lee, H. (2013). Product Development and Management Association's 2012 Comparative Performance Assessment Study. Journal of Product Innovation Management, 30(3): 408-429. https://doi.org/10.1111/jpim.12025

Moilanen, M., Østbye, S., \& Woll, K. (2014). Non-R\&D SMEs: external knowledge, absorptive capacity and product innovation. Small Business Economics, 43(2): 447-462. https://doi.org/10.1007/s11187-014-9545-9

Oficina Internacional del Trabajo. (2015). Informe IV. Pequeñas y medianas empresas y creación de empleo decente y productivo. In Conferencia Internacional del Trabajo, $104{ }^{a}$ reunión, 2015. Ginebra: OIT.

Organización de Cooperación y Desarrollo Económicos. (2015). Frascati Manual 2015: Guidelines for collecting and reporting data on research and experimental development. The Measurement of Scientific, Technological and Innovation Activities. Paris: OECD Publishing. https://doi.org/10.1787/9789264239012-en

Organización de Cooperación y Desarrollo Económicos, \& Oficina de Estadísticas de las Comunidades Europeas. (2005). Manual de Oslo. Guía para la recogida e interpretación de datos sobre innovación (3. ${ }^{\mathrm{a}}$ ed.). Madrid: Tragsa. https://doi.org/10.1787/9789264065659-es

Petti, C., \& Zhang, S. (2016). The role of absorptive capacity in Chinese firms. Measuring Business Excellence, 20(2): 1-12. https://doi.org/10.1108/MBE-11-2014-0047

Pulak, D., \& Bikash, B. (1997). Pay satisfaction of R\&D personnel in manufacturing organizations: the role of career comparision process. The Journal of High Technology Management Research, 8(2): 171-186. https://doi.org/10.1016/S1047-8310(97)90001-8Get

Qingqing, Z., Yanting, G., Mingtianzi, L., \& Gang, Z. (2016). Non-R\&D based innovation and the growth of SMEs in China: a case study of Hangzhou FC Company. In Kocaoglu, D. F. (Ed.), 2016 Proceedings of PICMET '16: Technology Management for Social Innovation (pp. 1065-1073). Portland: Portland State University.

Rammer, C., Czarnitzki, D., \& Spielkamp, A. (2009). Innovation success of non-R\&Dperformers: substituting technology by management in SMEs. Small Business Economics, 33(1), 35-58. https://doi.org/10.1007/s11187-009-9185-7

Rothwell, R. (1994). Towards the Fifth-generation Innovation Process. International Marketing Review, 11(1), 7-31. https://doi.org/10.1108/02651339410057491

Santamaría, L., Nieto, M. J., \& Barge-Gil, A. (2009a). ¿Hay innovación más allá de la I+D? El papel de otras actividades innovadoras. Universia Business Review, 22, 102-117.

Santamaría, L., Nieto, M. J., \& Barge-Gil, A. (2009b). Beyond formal R\&D: taking advantage of other sources of innovation in low- and medium-technology industries. Research Policy, 38(3), 507-517. https://doi.org/10.1016/j.respol.2008.10.004

Sempere-Ripoll, F., \& Hervás-Oliver, J. L. (2002). In times of economic crisis: innovation with, or without, R\&D activities? An analysis of Spanish companies. In Rüdiger, K., PerisOrtiz, M., \& Blanco-González, A. (Eds.), Entrepreneurship, innovation and economic 
crisis. Lessons for research, policy and practice (pp. 159-166). Springer Nature.

Sempere-Ripoll, F. S., \& Hervás-Oliver, J. L. (2011). ¿Qué explica la innovación en PyMEs? Dirección y Organización, 43, 5-15.

Siddharthan, N. S., \& Agarwal, R. N. (1992). Determinants of R\&D decisions : a crosssection study of Indian private corporate firms. Economics of Innovation and New Technology, 2(2): 103-110. https://doi.org/10.1080/10438599200000008

Som, O. (2012). Innovation without $R \& D$. Heterogeneous innovation patterns on non- $R \& D-$ performing firms in ther German manifacturing industry. Karlsruhe: Springer Gabler. https://doi.org/10.1007/978-3-8349-3492-5

Som, O., \& Kirner, E. (2015). Low-tech innovation. Competitiveness of the German manufacturing sector. Cham: Springer International Publishing.

Som, O., Kirner, E., \& Jäger, A. (2013). Absorptive capacity of non-R\&D-intensive firms in the German manufacturing industry. In 35th DRUID Celebration Conference 2013. Barcelona, Spain, June 17-19.

Sterlacchini, A. (1999). Do innovative activities matter to small firms in non-R\&D-intensive industries? An application to export performance. Research Policy, 28(8): 819-832. https://doi.org/10.1016/S0048-7333(99)00023-2

Trigo, A. (2013). The nature of innovation in R\&D- and non-R\&D-intensive service firms: evidence from firm-level latent class analysis. Industry and Innovation, 20(1), 48-68. https://doi.org/10.1080/13662716.2013.761380

Trujillo-Ruiz, F. B., Hervás-Oliver, J. L., \& Peris-Ortiz, M. (2015). Entrepreneurship and open innovation in Spanish manufacturing firms. In Peris-Ortiz, M., \& Sahut, J. M. (Eds.), New challenges in entrepreneurship and finance (pp. 247-258). Cham: Springer.

Ueki, Y. (2017). The roles of top management characteristics, human resource management and customer relationships in innovations: an exploratory analysis. Asian Journal of Technology Innovation, 25(2): 206-227. https://doi.org/10.1080/19761597.2017.1385962

Veugelers, R., \& Cassiman, B. (1998). Make and buy in innovation strategies: evidence from Belgian manufacturing firms. Research Policy, 28(1): 63-80. https://doi.org/10.1016/S0048-7333(98)00106-1

World Bank. (2017). Country profiles [dataset]. Data catalog. Washington D. C.: The World Bank Group. https://datacatalog.worldbank.org/dataset/country-profiles

Yanting, G., Xiao, C., \& Gang, Z. (2016). How Do Non-R \& D-based Innovations Affect SMEs ' Performance? The Mediating Role of Dynamic Capabilities. In Kocaoglu, D. F. (Ed.), 2016 Proceedings of PICMET '16: Technology Management for Social Innovation (pp. 879-886). Portland: Portland State University.

Zahra, S., \& George, G. (2002). Absorptive capacity: a review, reconceptualization, and extension. The Academy of Management Review, 27(2): 185-203. https://doi.org/10.2307/4134351. 